Supporting Information

\title{
Esterase-responsive polypeptide vesicles as sustained-release nanocompartments for fibroblast-exempt drug delivery
}

Weihua Duan, ${ }^{a}$ Sifan $J i,{ }^{a}$ Yu Guan, ${ }^{a}$ Xueluer Mu, ${ }^{a}$ Sha Fang, ${ }^{a}$ Yingxi Lu, ${ }^{b}$ Xianfeng

Zhou, ${ }^{a,}{ }^{*}$ Jing Sun, ${ }^{a}$ Zhibo Li. ${ }^{a}{ }^{*}$

a Key Lab of Biobased Polymer Materials of Shandong Provincial Education Department, College of Polymer Science and Engineering, Qingdao University of Science and Technology, 53 Zhengzhou Road, Qingdao, 266042, P.R. China.

${ }^{\mathrm{b}}$ College of Material Science and Engineering, Qingdao University of Science and Technology, Qingdao, 266042, P.R. China.

${ }^{*}$ To whom correspondence should be addressed.

E-mail: xianfeng@qust.edu.cn; zbli@qust.edu.cn

Phone: $+86-532-84022950$ 
Synthetic Methods:

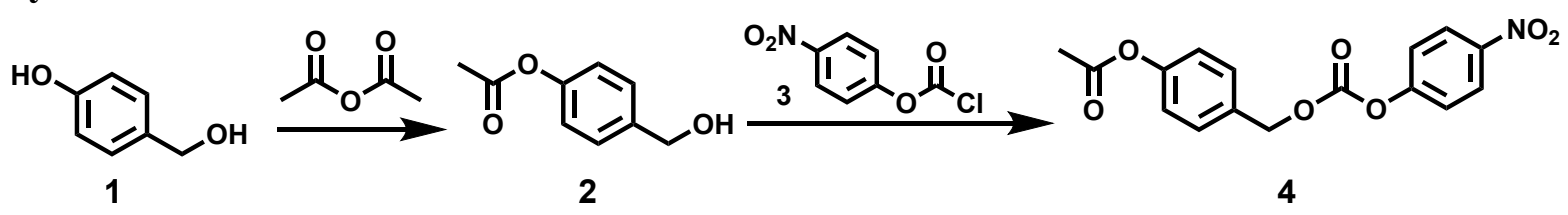

Scheme S1. The synthetic route of Compound 4

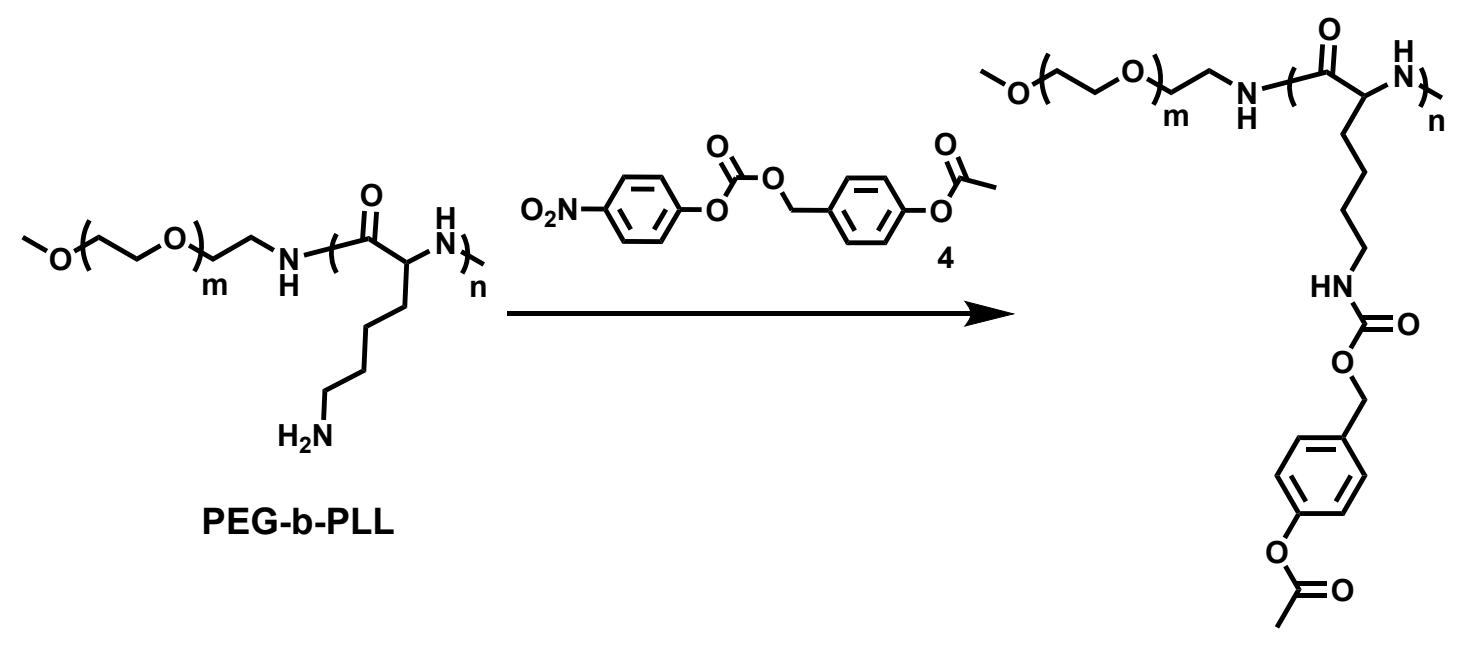

PEG-b-PLLNA

Scheme S2. The synthetic route of PEG- $b$-PLLNA copolypeptide using the post-modification method.

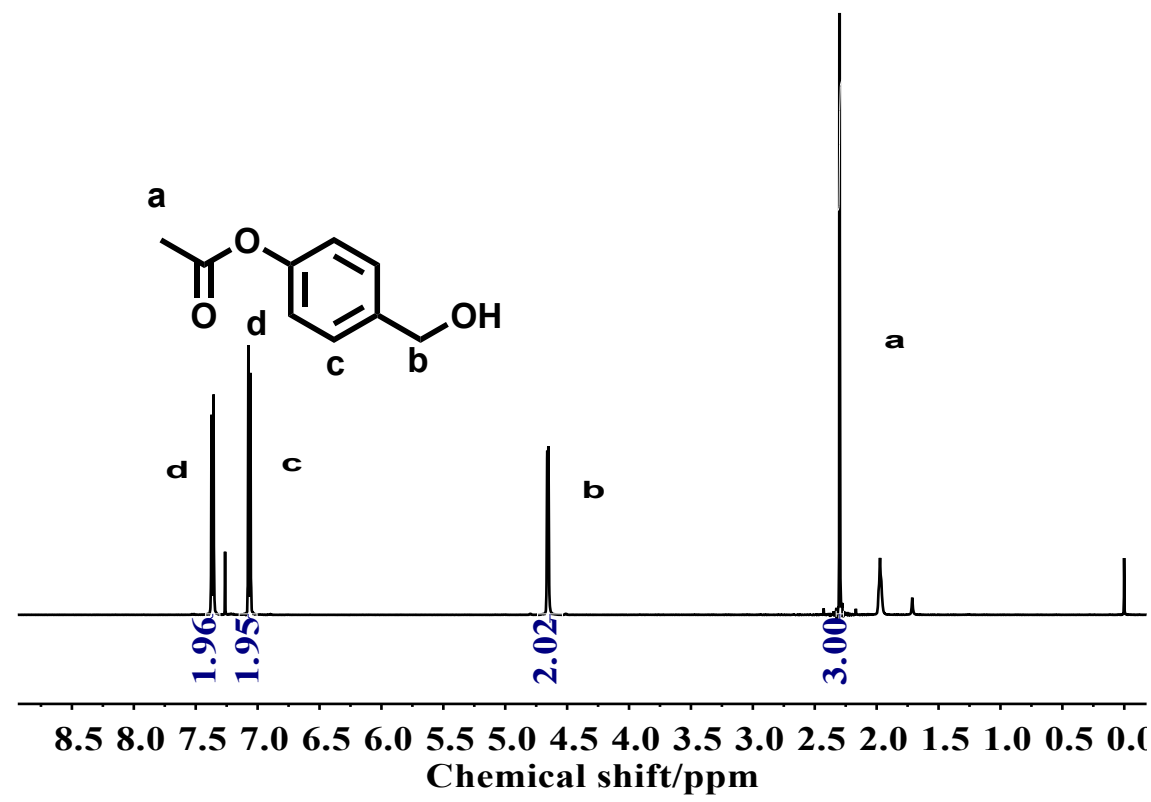

Figure S1. ${ }^{1} \mathrm{H}$ NMR spectrum of compound 2 in $\mathrm{CDCl}_{3}$ at $298 \mathrm{~K}$. 


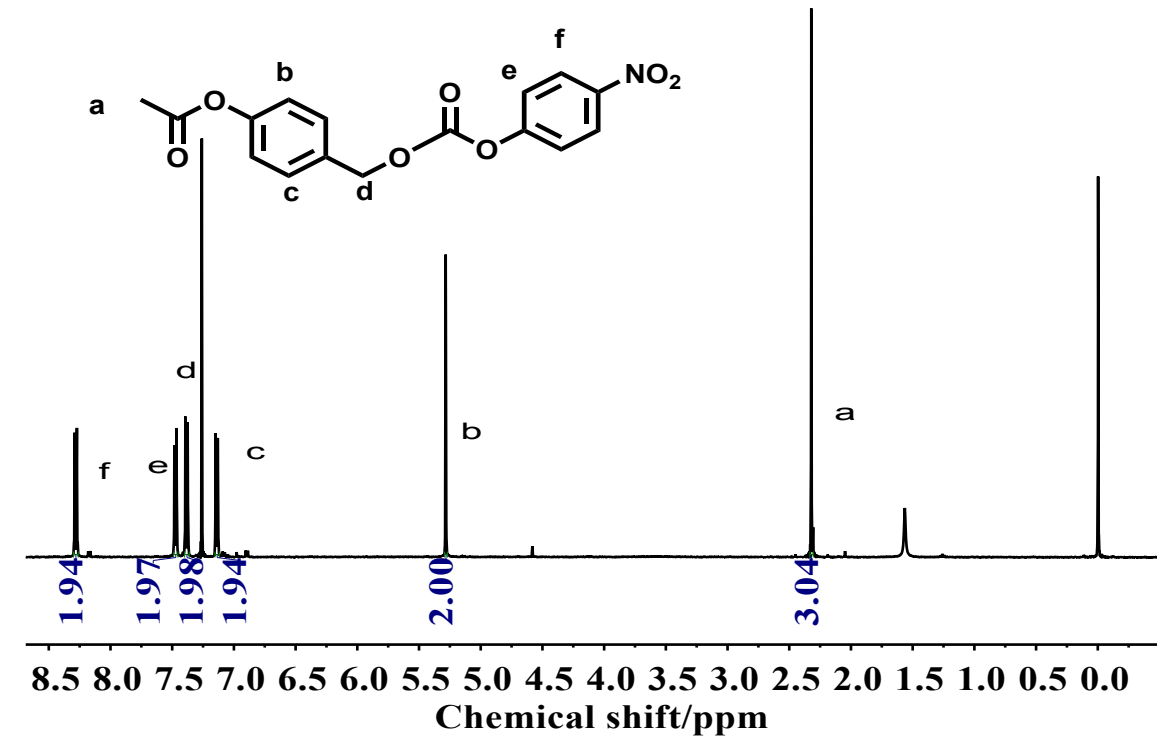

Figure S2. ${ }^{1} \mathrm{H}$ NMR spectrum of compound 4 in $\mathrm{CDCl}_{3}$ at $298 \mathrm{~K}$.

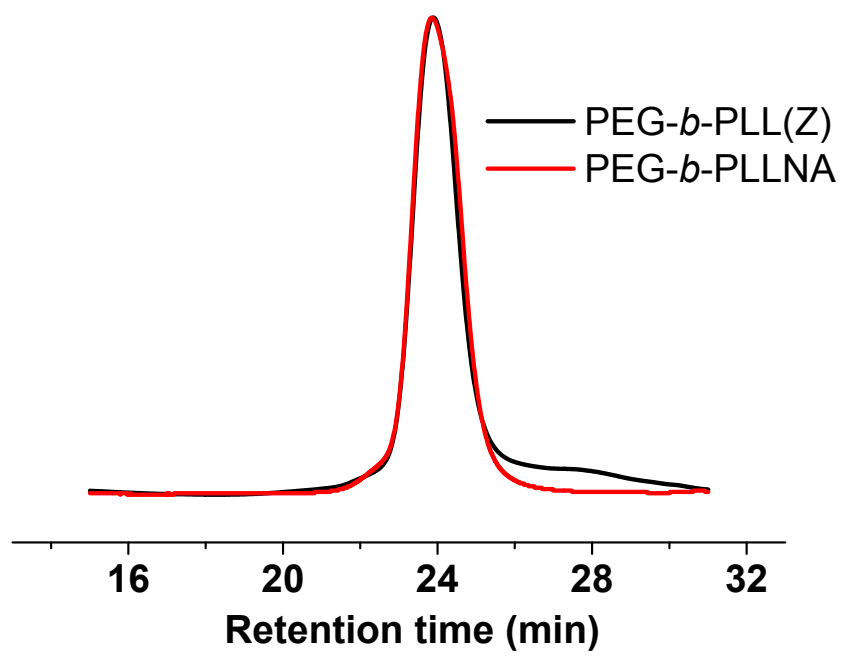

Figure S3. SEC traces of PEG-b-PLL(Z) and PEG- $b$-PLLNA. DMF was used as the eluent at a flow rate of $1.0 \mathrm{~mL} / \mathrm{min}$ at $50{ }^{\circ} \mathrm{C}$. 


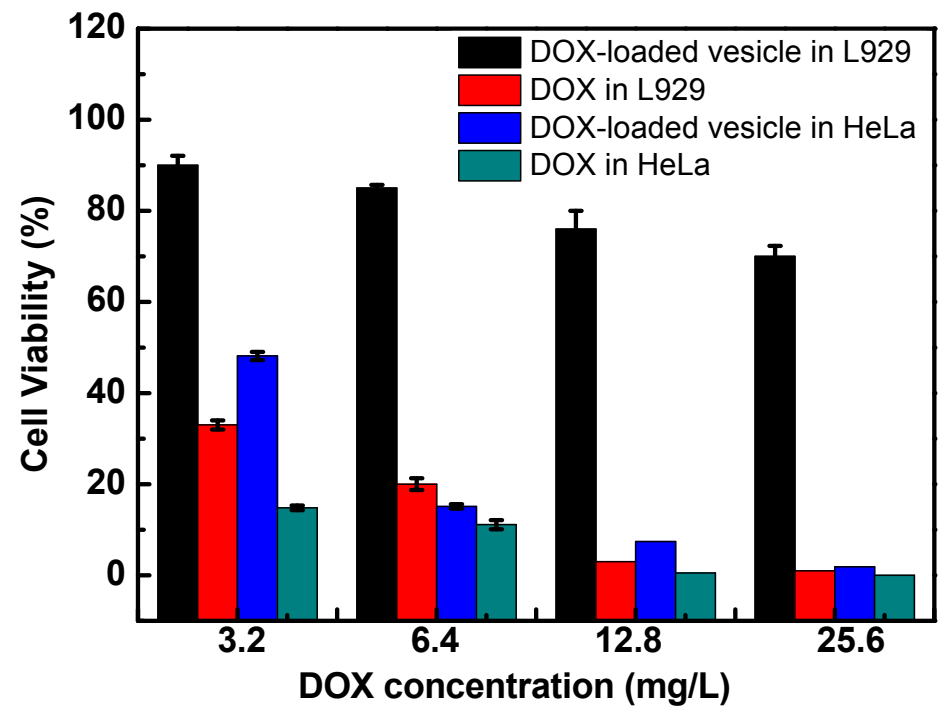

Figure S4. In Vitro cytotoxicity of DOX-loaded polypeptide vesicles to both L929 fibroblast and HeLa cancer cells, with equal concentration of free DOX as control. 